\title{
A Comparison of Antioxidant Properties in Organic and Conventional Blueberries
}

\author{
Gaytri Gupta-Elera ${ }^{1}$, Andrew Garrett ${ }^{1}$, Andres Martinez ${ }^{1}$, Ryan D. Kraus ${ }^{1}$, Richard Robison ${ }^{1} \&$ Kim O’Neill ${ }^{1}$ \\ ${ }^{1}$ Department of Microbiology and Molecular, Brigham Young University, Utah, USA \\ Correspondence: Kim L. O’Neill, Brigham Young University, Provo, UT 84604, USA. Tel: 1-801-422-2449. \\ E-mail:kim_oneill@byu.edu
}

Received: April 6, 2012 Accepted: April 23, 2012 Online Published: May 28, 2012

doi:10.5539/jfr.v1n3p1 URL: http://dx.doi.org/10.5539/jfr.v1n3p1

\begin{abstract}
Vacciniumcyanococcus, most commonly known as blueberry, is a fruit native to North America that is known for its unique taste and high antioxidant content. The skin, seed and juice of both organically and conventionally grown blueberry extract were analyzed for antioxidant content using both the Hydrophilic and Lipophilic Oxygen Radical Absorbance Capacity (ORAC) assays. Results from the Hydrophilic ORAC test showed that conventionally grown blueberries had a higher antioxidant capacity across all samples, while the Lipophilic ORAC assay showed that the antioxidant concentration of organically cultivated blueberry juice was highest, but conventionally grown blueberry seed and skin extract showed higher antioxidant content. The vitamin $\mathrm{C}$ content of both conventional and organic blueberries was analyzed using High Performance Liquid Chromatography (HPLC), where the organic blueberries showed a higher vitamin C concentration. In general, both organic and conventional blueberries are rich in antioxidants, and therefore, possess potential health benefits that require further study.
\end{abstract}

Keywords: blueberry, antioxidant, organic, conventional, ORAC

\section{Abbreviations}

AAPH: 2, 2'-azobis (2-aminidopropane) dihydrochloride

ORAC: oxygen radical absorbance capacity

ROS: Reactive Oxygen Species

TE: Trolox Equivalents

AUC: area under the curve

\section{Introduction}

In recent years, there has been a rising interest in organically cultivated foods as an alternative to conventional agricultural techniques, which has led to an increase in the acreage assigned to organic cultivations (Carbonaro, Mattera, Nicoili, Bergamo, \& Cappeloni, 2002). Differences between these farming techniques are attributed to fertilizer type and pesticide use. Conventional farming techniques involve the use of mineral fertilizers and synthetic pesticides, while organic farming techniques use organic fertilizers and no synthetic pesticides.

Whether it is more beneficial for the consumer to purchase organic instead of conventional foods remains a hot topic of discussion. Although most people are under the impression that organically cultivated foods are more beneficial (Worthington, 2004), the experimental evidence to support this claim remains unclear, and assessment of the health benefits of these products still requires further investigation.

Interest in the consumption of foods with the greatest health benefit potential has become more prevalent because epidemiological studies suggest that the consumption of both an antioxidant and phytochemical-rich diet contribute to the reduction of heart disease and several types of cancer (Chinery et al., 1998).

As a result, there has been a substantial amount of interest in Vacciniumcorymbosum, commonly known as blueberries, due to this fruit's anti-tumor properties in different cancer types such as colon cancer (Yi, Fisher, Krewer, \& Akoh, 2005), breast cancer (Adams et al., 2010), and prostate cancer (Schmidt, Erdman, \& Lila, 2006). Blueberries are native to North America, and are known to contain high contents of both antioxidants, such as phenolic acid and resveratrol, and phytochemicals, such as flavonoids and tannins (Seeram, 2008). 
Antioxidants provide protection against oxidative stress, a condition that has been suggested to contribute to a wide variety of diseases, including artherosclerosis, Parkinson's disease, and cancer (Amira, 2010). Oxidative stress occurs when reactive oxygen species (ROS) and reactive nitrogen species (RNS) increase to levels that propagate oxidative injury to DNA, lipids and proteins. Damage to DNA is an early event in carcinogenesis, and oxidation is known to induce 2'-4'-oxidative DNA damage that may lead to the formation of DNA-protein cross-links, alterations in the carbohydrate-phosphate backbone, or direct modification of purine or pyrimidine bases. If these alterations lead to the activation of oncogenes or the inactivation of tumor suppressor genes, cancer may develop (Gupta-Elera, Garrett, Martinez, Robison, \& O'Neill, 2010). Therefore, antioxidants such as phenolic acid protect cells against oxidative stress by scavenging oxygen free radicals, and functioning as metal chelators (Russo et al., 2000). Resveratrol, another antioxidant found in blueberries, additionally reduces levels of ROS by increasing the levels of MnSOD, an enzyme that reduces superoxide to hydrogen peroxide (Robb, Page, Wiens, \& Stuart, 2008), and also interferes with carcinogenesis by inducing Fas/Fas ligand-mediated apoptosis (Cao, Fu, Wang, Liu, \& Han, 2005).

Phytochemicals have both antioxidant and hormone mimicking actions, and they exert their anticancer effects through different mechanisms, such as the induction of metabolizing enzymes, alteration of gene expression, modulation of cell signaling pathways and DNA repair (Seeram, 2008). Phytochemicals in blueberries have been shown to decrease the secretion of urokinase-type plasminogen activator (uPA) and increase the secretion of plasminogen activator inhibitor (PAI), both of which are cell-signaling proteins that inhibit tumor cell proliferation and metastatic potential in breast cancer cells (Adams et al., 2010). Anthocyanin, the flavonoid responsible for the pigmentation in blueberries, has both antioxidant and anti-cancer properties (Seeram, Yanjun, \& Muraleedharan, 2003). A study that explored the potential anticancer activities of polyphenols in rabbiteye blueberries on HepG2 liver cancer cells found that the anthocyanin fraction had the greatest inhibitory effect with 50\%inhibition of cancer cell growth, thus suggesting that blueberries may help reduce the risk of liver cancer (Yi, Akoh, Fischer, \& Krewer, 2006).

The main objective of this research was to provide preliminary results on the differences in antioxidant concentrations between conventionally and organically grown blueberries, specifically by examining extracts of their juice, skin and seed; and, therefore, provide insight into the health benefits of organic vs. conventional blueberries.

\section{Materials and Methods}

\subsection{Chemicals}

The 2,2'-azobis(2-aminidopropane) dihydrochloride (AAPH) was purchased from Wako Chemicals USA, Inc. (Richmond, VA). Fluorescein-sodium salt, Metaphosphoric Acid, methylated $\beta$-cyclodextrin, and DL-Dithiothreitol (threo-1,4-dimercapto-2,3-butanediol) were obtained from Sigma-Aldrich, Inc. (Milwaukee, WI). Trolox (6-hydroxy-2,5,7,8-tetramethyl-2-carboxylic acid) was purchased from Enzo Life Sciences, Inc. (Plymouth, PA). Acetonitrile, methanol, and acetone were purchased from Baxter (Muskegon, MI).

\subsection{Materials}

Costar 96-well plate and 24-well clear culture plates were purchased from Corning Inc. (Lowell, MA).

\subsection{Equipment}

All fluorescence readings were taken using the BMG FLUOstar Optima (BMG Laboratories) microplate reader (serial \#413-0225). HPLC readings were made using the Agilent 1100 Zorbax SB-Aq (serial \# USVD001136). Data were analyzed using Microsoft Excel and Apple Numbers.

\subsection{Fruit Sample}

Ten different lots of both conventional and organic highbush blueberry fruits were purchased at a local grocery store in Provo, UT on the day of importation. Organic blueberries carried the USDA organic label. Both organic and conventional blueberries were grown in Michigan.

\subsection{Sample preparation}

Blueberry skin, seed and juice were isolated and were then homogenized by weighing $0.1 \mathrm{~g}$ of sample and diluting it in $10 \mathrm{ml}$ of double distilled water. Samples were centrifuged at $1800 \mathrm{rpm}$ for 15 minutes, placed in aliquots of $2.5 \mathrm{ml}$ and kept in a freezer at $-20^{\circ} \mathrm{C}$ until analyzed.

\subsection{Hydrophilic and Lipophilic ORAC}

All samples were analyzed using a modified ORAC assay based on published methods (Garrett, Murray, 
Robison, \& O'Neill, 2010; Huang, 2002). The ORAC assay measures oxidative degradation of a fluorescent compound (fluorescein sodium salt) after being exposed to an oxygen radical initiator, AAPH (2,2'-azobis-(2-amidinopropane) dihydrochloride). All readings were taken in Cole-Parmer black bottom 96-well plates, with $200 \mu$ Fluorescein, $25 \mu \mathrm{AAPH}$, and $20 \mu \mathrm{l}$ of antioxidant sample or PBS in each well.

The Lipophilic ORAC was performed in duplicate as described for the Hydrophilic ORAC, except that the solvent used to dilute the sample was $7 \%$ randomly methylated- cyclodextrin (RMCD) in acetone/water $(1: 1 \mathrm{v} / \mathrm{v})$ solution.

Readings were taken every 2 minutes for 90 minutes. Measurements were taken by the BMG FLUOstar Microplate reader at $37^{\circ} \mathrm{C} ; 485 \mathrm{~nm}$ excitation and $590 \mathrm{~nm}$ emission filters were used for fluorescence readings. All fluorescent measurements were expressed relative to the initial reading. The area under the curve (AUC) was calculated by using the difference of area under the fluorescein decay curves between the sample curve and the fluorescein + AAPH curve. The AUC measures the total antioxidant activity in the sample minus the fluorescein + AAPH antioxidant curve. Lipophilic and Hydrophilic ORAC values were measured separately and the Total Antioxidant Capacity (TAC) was calculated by summing the L-ORAC and H-ORAC values.

\subsection{Standard Curve Data}

Trolox, a water-soluble analogue of vitamin E, was used as a control standard. Trolox standards were run at concentrations of $50 \mu \mathrm{M}, 25 \mu \mathrm{M}, 12.5 \mu \mathrm{M}$, and $6.25 \mu \mathrm{M}$ to obtain standard curves for each trial. Data from each standard curve was used to convert raw net area under the sample curve (net AUC) values to Trolox Equivalents per liter (TE/L). Subsequent calculations converted TE/L to TE/100g (see Figure A.1)

\subsection{High-Performance Liquid Chromatography}

All samples were measured using an Agilent 1100 (US) HPLC system coupled with an Agilent 1100 diode array detector and equipped with Agilent 1100 binary pumps based on published methods (Nielson, 2006). Samples consisting of $5 \mu \mathrm{L}$ were injected at ambient temperature $\left(20^{\circ} \mathrm{C}\right)$ into a Reverse phase Zorbax SB-Aq (4.6x150 $\mathrm{mm} 3.5 \mu \mathrm{m}$ ) with $\mathrm{C} 18$ guard column. An Isocratic mobile phase consisting of MeOH/Sodium Acetate (3/97) with a pH of 4.0 was used with a flow rate of $1 \mathrm{~mL} / \mathrm{min}$. Vitamin C content was identified by its UV spectra, recorded with a diode array detector and by chromatographic comparison with authentic markers. Scanning between 190 and $400 \mathrm{~nm}$ was performed, and data were collected by the Agilent Chemstation data system. Data collection time was 30 minutes.

\section{Results}

\subsection{Hydrophilic ORAC Values}

As shown in Figure 1, hydrophilic antioxidant analysis of conventional blueberry skin, seed and juice extract resulted in higher antioxidant values when compared to the organic blueberries, across all samples. Juice exhibited the highest measured antioxidant activity, with the conventional yielding a value of $9,806 \mathrm{TE} / 100 \mathrm{~g}$ ( $\operatorname{stdev}=3851, \mathrm{~N}=30$ ), and the organic yielding a value of 5,258 TE/100g ( $\mathrm{stdev}=2649, \mathrm{~N}=30$ ). The next highest activity was found in the skin at 7,891 TE/100g ( $\operatorname{stdev}=2311, \mathrm{~N}=30)$ for conventional, and at 4,623 TE/100g $(\operatorname{stdev}=2337, \mathrm{~N}=30)$ for organic. Finally, the lowest activity was measured in the seed, with the conventional at $1,015 \mathrm{TE} / 100 \mathrm{~g}(\mathrm{stdev}=524, \mathrm{~N}=30)$, and the organic at $636 \mathrm{TE} / 100 \mathrm{~g}(\mathrm{stdev}=283, \mathrm{~N}=30)$.

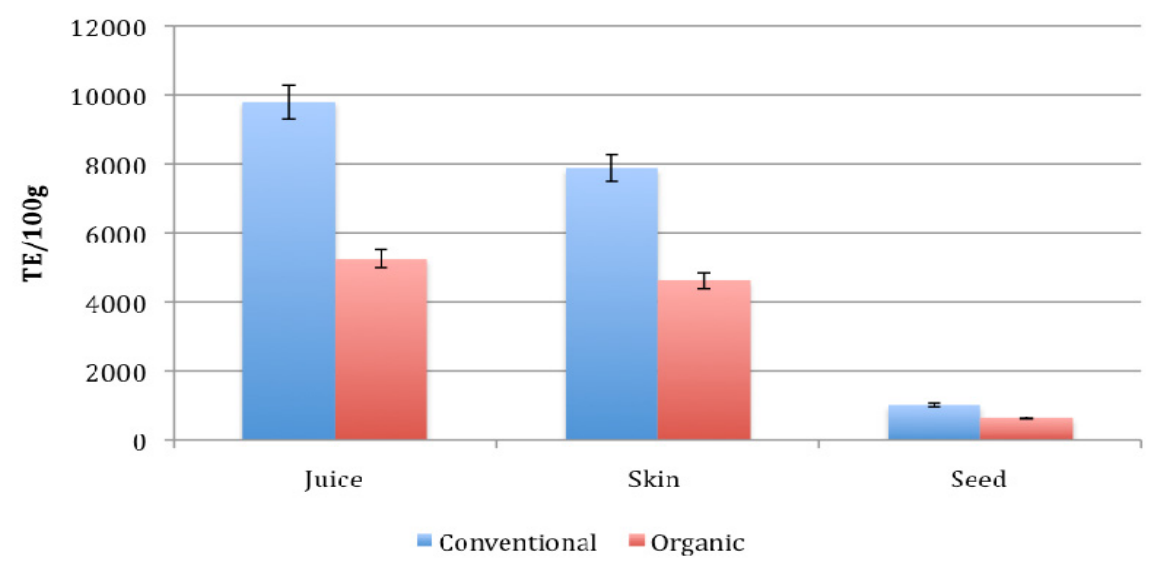

Figure 1. Differences in antioxidant content between organic and conventional blueberry juice, skin, and seed extract using H-ORAC. All values are reported as TE/100g (Mean of $\mathrm{N}=30$ ) 


\subsection{Lipophilic ORAC Values}

As shown in Figure 2, lipophilic antioxidant analysis of the conventional blueberry skin and seed extract resulted in a higher antioxidant content in comparison to the organic blueberry samples, while the organic juice extract resulted in a higher antioxidant content than that of the conventional. The highest measured antioxidant activity was found in the juice, with the organic yielding a value of 35,156 TE/L (stdev=2,183, N=30), and the conventional yielding a value of $31,535 \mathrm{TE} / \mathrm{L}(\operatorname{stdev}=2,281, \mathrm{~N}=30)$. The next highest activity was observed in the skin at 13,755 TE/100g ( $\operatorname{stdev}=2,593, \mathrm{~N}=30)$ for conventional, and at 11,833 TE/100g ( $\mathrm{stdev}=1,687, \mathrm{~N}=30$ ) for organic. Finally, the lowest activity was found in the seed, with the conventional at $10,727 \mathrm{TE} / 100 \mathrm{~g}$ $($ stdev $=2,198, \mathrm{~N}=30)$, and the organic at $8,709 \mathrm{TE} / \mathrm{L}(\operatorname{stdev}=1,830, \mathrm{~N}=30)$.

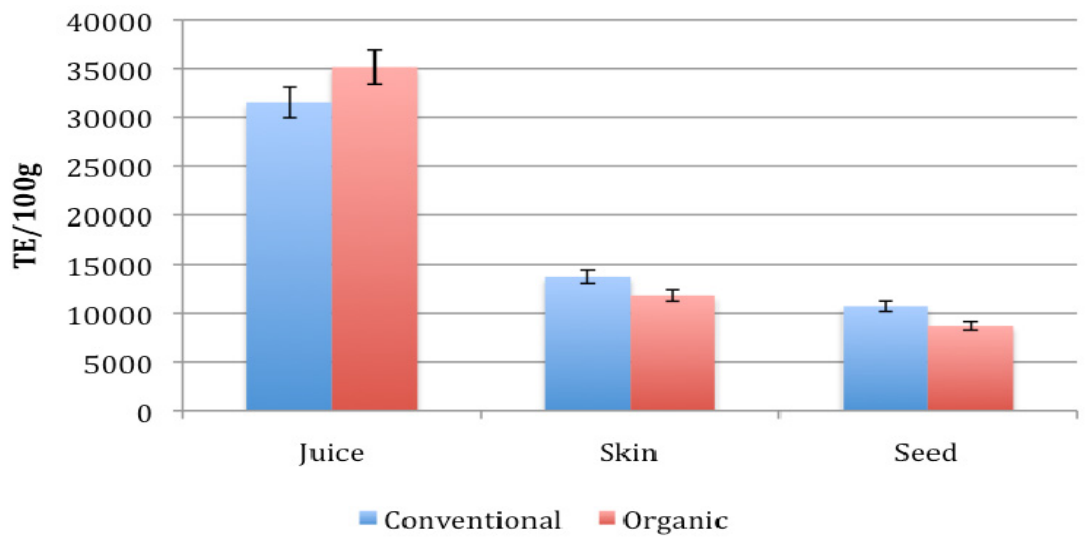

Figure 2. Differences in antioxidant content between organic and conventional blueberry juice, skin, and seed extract using L-ORAC. All values are reported as TE/100g (Mean of $\mathrm{N}=30$ )

\subsection{Vitamin C content using HPLC}

High-Performance Liquid Chromatography analysis of whole organic blueberries resulted in a higher vitamin $\mathrm{C}$ content than whole conventional blueberries, as shown in Figure 3. Organic blueberries yielded a value of $592.879 \mu \mathrm{g} / \mathrm{g}(\mathrm{stdev}=42.406, \mathrm{~N}=5)$, while conventional blueberries had $386.947 \mu \mathrm{g} / \mathrm{g}(\mathrm{stdev}=40.172, \mathrm{~N}=5)$.

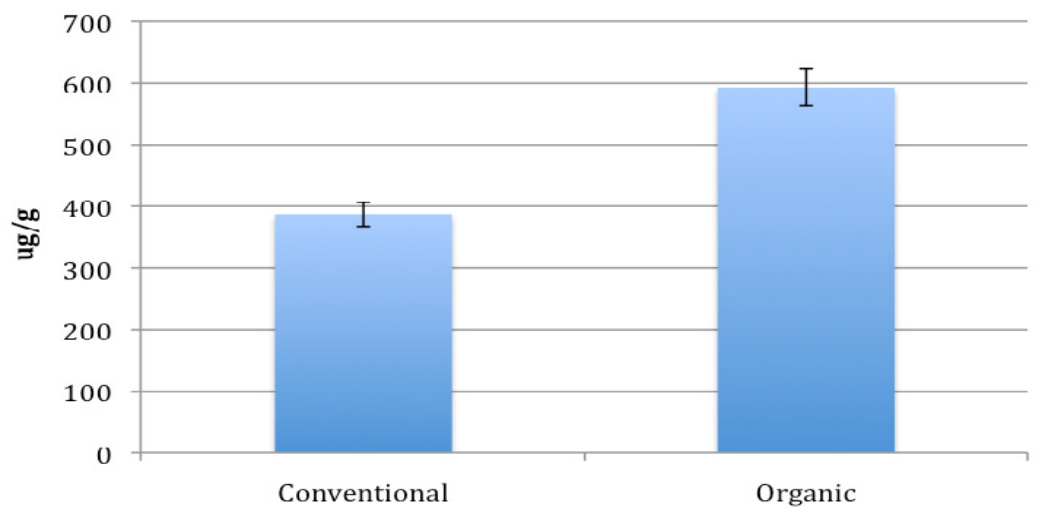

Figure 3. Difference in vitamin C content between organic and conventional blueberries using HPLC. All values are reported as $\mu \mathrm{g} / \mathrm{g}$

\section{Discussion}

\subsection{Analysis of H-ORAC and L-ORAC values}

When the extracts of blueberry seed, skin and juice were analyzed for antioxidant activity using the Hydrophilic ORAC, conventionally grown blueberries exhibited higher antioxidant content across all samples. Statistical significant changes in antioxidant content were observed in blueberry skin $(p<0.01)$, seed $(p<0.05)$, and juice 
$(\mathrm{p}<0.01)$ components, with conventional samples possessing more antioxidant activity. This might be explained in part by the difference in nutrient composition between organic and mineral fertilizers. Organic fertilizers have a lower nutrient content, solubility and nutrient release rates, thus making the nutrients, such as nitrogen, less readily available to plants. Therefore, the blueberry plant grown in an organic environment has to allocate more of its resources towards the synthesis of nitrogen-containing compounds and towards its growth and development, whereas conventionally grown blueberries can allocate their resources elsewhere (Seghers et al., 2003).

The Lipophilic ORAC provided different results. The extract of organic blueberry juice showed a statistically significant increase $(\mathrm{p}<0.001)$ in antioxidant concentration when compared to conventional blueberry juice. On the other hand, conventionally grown blueberries showed a statistically significant increase of antioxidants in their skin $(p<0.001)$ and seed $(p<0.001)$, when compared to its organic blueberry components. Winter \& Davis (2006) have suggested that a potential reason that could account for the elevated antioxidant content in organic blueberry juice is that since organically grown blueberries do not have synthetic pesticides, the plant has to build its own chemical defenses against pathogens, thus increasing their antioxidant concentrations. However, they also argued that conventionally-grown blueberry plants are also subjected to stress due to their exposure to sub-lethal doses of chemicals found in both herbicides and pesticides, and in response, they increase their antioxidants and phytochemicals so that they can act as chemical barriers against pathogens (Winter \& Davis, 2006).

\subsection{Analysis of Vitamin C content using HPLC}

Results showed that organically grown blueberries had a $53.4 \%$ antioxidant increase when compared to conventionally grown blueberries. Ascorbic acid reduces reactive oxygen species to prevent oxidative damage, and also reduces vitamin $\mathrm{E}$ to activate it and restore the antioxidant protection of the cell membrane (O'Neill, Standage, Hughes, \& Murray, 2001). The decreased amount of vitamin C in conventionally grown blueberries could be due to the synthetic fertilizer. The amount of nitrogen in the fertilizer might affect the amount of vitamin $\mathrm{C}$ and nitrates, and the amount of protein produced by the plant. Since plants that are conventionally grown are presented with a higher concentration of nitrogen, its protein production is increased, while its carbohydrate production is reduced. Consequently, the amount of vitamin $\mathrm{C}$ produced is reduced, since it is made from carbohydrates (Seghers et al., 2003).

This is consistent with results from a review that surveyed 41 studies in the existing literature comparing the nutritional content of organic and conventional crops in order to identify significant differences; and trends in the data found that, on average, the vitamin $\mathrm{C}$ content of an organic fruit or vegetable was $27 \%$ higher than that of a conventional fruit or vegetable (Worthington, 2004).

Student's one tail t-test $\left(6.69 \mathrm{e}^{-30}\right)$ and two tail t-test $\left(1.34 \mathrm{e}^{-29}\right)$ show that there is a statistically significant difference in vitamin $\mathrm{C}$ capacity between organic and conventional blueberries.

\subsection{Analysis of total ORAC values}

As shown in Figure 4, when the total antioxidant capacity for the H-ORAC and L-ORAC are analyzed, conventional blueberries have a higher total antioxidant content, with 33,388 TE/100g and 607,700 TE/L, respectively, in comparison to $25,800 \mathrm{TE} / 100 \mathrm{~g}$ and $415,608 \mathrm{TE} / \mathrm{L}$ for organic blueberries. Studen's t-test analyses performed on data revealed that the differences in antioxidant content of juice, skin and seed between organically- and conventionally-grown blueberries were statistically significant: $\mathrm{p}<0.01, \mathrm{p}<0.01$, and $\mathrm{p}<0.01$, respectively.

Overall, the blueberry fruit is very rich in antioxidants, with ORAC values higher than blackberries, strawberries and grapes (Haytowitz \& Bhagwat, 2010). A study that analyzed the flavonoid and phenolic acid profiles of 19 different berries, including cranberry, raspberry and strawberry, suggested that blueberries had the highest total phenolic content (Häkkinena et al., 1999). This suggests that the potential health benefits of blueberries is high, and may, thereby, prevent the initiation and progression of diseases that have been linked to oxidative stress, such as cancer, cardiovascular diseases and neurodegenerative diseases. 


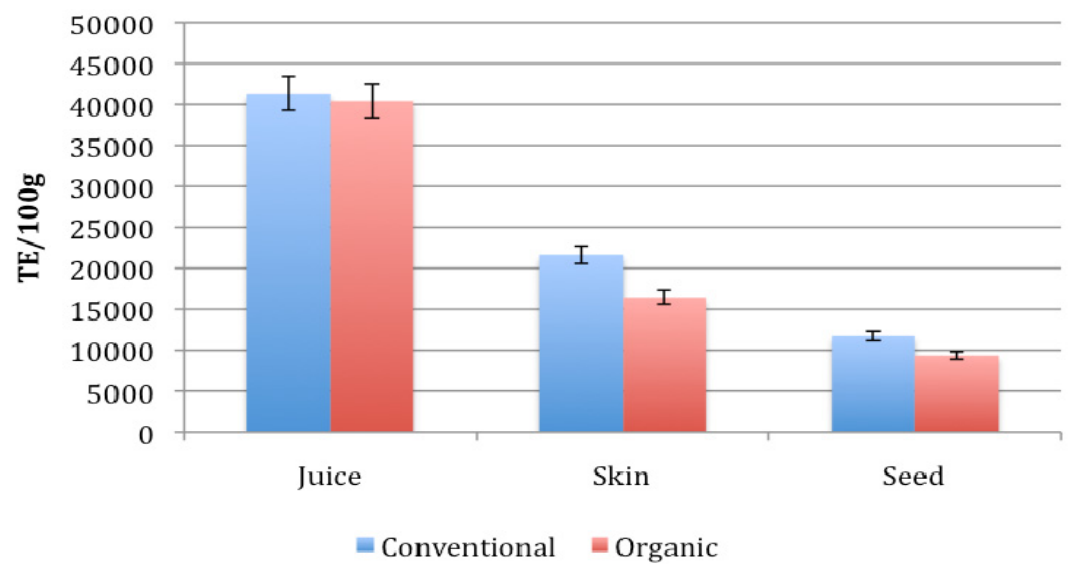

Figure 4. Differences in total antioxidant content between organic and conventional blueberry juice, skin, and seed extract. All values are reported as TE/100g (Mean of $\mathrm{N}=5)$

\section{Conclusion}

Results showed that although organic and conventional blueberries are both very rich in antioxidants, conventional blueberries have higher antioxidant content, and, therefore, may have a higher overall health benefit potential in reducing cancer risk. However, organic blueberries have higher vitamin $\mathrm{C}$ content when analyzed with HPLC. This study also suggests that it is important to consume both the juice and skin of the fruit to maximize antioxidant intake.

Further chemical analyses are needed to more fully understand the chemical composition in these blueberry components and the specific antioxidant compounds they contain. Further research may also include establishing a cellular model, where different cancer cell lines can be exposed to both organic and conventional blueberry juice, skin and seed extract where the concentration of antioxidants that is absorbed by the cell can be measured. This may provide insight into how effectively the cells take in the antioxidants, and ultimately, add insight into the potential role of blueberries in the prevention of different cancer types.

\section{References}

Adams, L. S., Phung, S., Yee, N., Seeram,N., Li, L., \& Chen, S. (2010). Blueberry Phytochemicals Inhibit Growth and Metastatic Potential of MDA-MB-231 Breast Cancer Cells through Modulation of the Phosphatidylinositol 3-Kinase Pathway. Cancer Research, 70(9), 3594-3605. http://dx.doi.org/10.1158/0008-5472.CAN-09-3565

Amira, A. (2010). Oxidative Stress and Disease: An Updated Review. Research Journal of Immunology, 3(2), 129-145. http://dx.doi.org/10.3923/rji.2010.129.145

Cao, Y., Fu, Z., Wang, F., Liu, H., \&Han, R. (2005). Anti-angiogenic activity of resveratrol, a natural compound from medicinal plants. Journal of Asian natural products research, 7(3), $205-213$. http://dx.doi.org/10.1080/10286020410001690190

Carbonaro, M., Mattera, M., Nicoili, S., Bergamo, P., \& Cappeloni, M. (2002). Modulation of Antioxidant Compounds in Organic vs Conventional Fruit (Peach, Prunus persica L., and Pear, Pyrus communis L.). Journal of Agricultural and Food Chemistry, 50(19), 5458-5462.

Chinery, R. B., R.D., Shyr, Y., Kirkland, R., Coffey, R. J., \& Morrow, J. D. (1998). Antioxidants Reduce Cyclooxygenase-2 Expression, Prostaglandin Production, and Proliferation in Colorectal Cancer Cells. Cancer Research, 58(11), 2323-2327.

Garrett, A., Murray, B. K., Robison, R. A., \& O'Neill, K. L. (2010). Measuring antioxidant capacity using the orac and tosc assays. Methods in Molecular Biology, 594, $251-262$. http://dx.doi.org/10.1007/978-1-60761-411-1_17

Gupta-Elera, G., Garrett, A., Martinez, A., Robison, R., \& O'Neill, K. L. (2010). The antioxidant properties of the cherimoya (Annona cherimola) fruit. Food Research International, 44(7), $2205-2209$. http://dx.doi.org/10.1016/j.foodres.2010.10.038 
Häkkinena, S., Heinonenc, M., Kärenlampid, S., Mykkänena, H., Ruuskanene, J., \& Törrönen, R. (1999). Screening of selected flavonoids and phenolic acids in 19 berries. Food Research International, 32(5), 345-353. http://dx.doi.org/10.1016/S0963-9969(99)00095-2

Haytowitz, D., \& Bhagwat, S. (2010). USDA Database for the Oxygen Radical Capacity (Orac) of Selected Foods. From USDA National Nutrient Database for Standard Reference.

Huang, D. (2002). Development and Validation of Oxygen Radical Absorbance Capacity Assay for Lipophilic Antioxidants Using Randomly Methylated-Cyclodextrin as the Solubility Enhancer. Journal of Agricultural and Food Chemistry, 50(7), 1815-1821.

Nielson, A. P., Ogden, L. V., \& Pike, O. A. (2006). Sensory and Nutritional Quality of Dehydrated Potato Flakes in Long-Term Storage. Journal of Food Science, 71(6), 461-466. http://dx.doi.org/10.1111/j.1750-3841.2006.00084.x

O'Neill, K. L., Standage, S., Hughes, B., \& Murray, B. (2001). Fruits and Vegetables and the Prevention of Oxidative DNA Damage. In R. Watson (Ed.), Vegetables, Fruits, and Herbs in Health Promotion, pp. 135-143. http://dx.doi.org/10.1201/9781420042542.ch10

Robb, E., Page, M. M., Wiens, B. E., \& Stuart, J. (2008). Molecular mechanisms of oxidative stress resistance induced by resveratrol: Specific and progressive induction of MnSOD. Biochemical and Biophysical Research Communications, 367(2), 406-412. http://dx.doi.org/10.1016/j.bbrc.2007.12.138

Russo, A., Acquaviva, R., Campisi, A., Sorrenti, V., Di Giacomo, C., Virgata, G., Barcellona, M., \& Vanella, A. (2000). Bioflavonoids as antiradicals, antioxidants and DNA cleavage protectors. Cell Biology and Toxicology, 16(8), 91-98. http://dx.doi.org/10.1023/A:1007685909018

Schmidt, B. M., Erdman, J. W., \& Lila, M. A. (2006). Differential effects of blueberry proanthocyanidins on androgen sensitive and insensitive human prostate cancer cell lines. Cancer Letters, 231(2), $240-246$. http://dx.doi.org/10.1016/j.canlet.2005.02.003

Seeram, N. (2008). Berry Fruits: Compositional Elements, Biochemical Activities, and the Impact of Their Intake on Human Health, Performance, and Disease. Journal of Agricultural and Food Chemistry, 56, 627-629. http://dx.doi.org/10.1021/jf071988k

Seeram, N., Yanjun, Z., \& Muraleedharan, N. G. (2003). Inhibition of proliferation of human cancer cell lines and cyclooxygenase enzymes by anthocyanidins and catechins. Nutrition and Cancer, 46(1), 101-106. http://dx.doi.org/10.1207/S15327914NC4601_13

Seghers, D., Top, E.M., Reheul, D., Bulcke, R., Boeckx, P., Verstraete, W., \& Siciliano, S. D. (2003). Long-term effects of mineral versus organic fertilizers on activity and structure of the methanotrophic community in agricultural soils. Environmental Microbiology, 5(10), 867-877.

Winter, C. K., \& Davis, S. F. (2006). Organic Foods. Journal of Food Science, 71(9), $117-124$. http://dx.doi.org/10.1111/j.1750-3841.2006.00196.x

Worthington, V. (2004). Nutritional quality of organic versus conventional fruits, vegetables, and grains. Journal of Alternative and Complementary $\quad$ Medicine, $\quad 7(2), \quad$ 161-173. http://dx.doi.org/10.1089/107555301750164244

Yi, W., Akoh, C., Fischer, J., \& Krewer, G. (2006). Effects of phenolic compounds in blueberries and muscadine grapes on HepG2 cell viability and apoptosis. Food Research International, 39(5), 628-638. http://dx.doi.org/10.1016/j.foodres.2006.01.001

Yi, W., Fisher, J., Krewer, G., \& Akoh, C. (2005). Phenolic Compounds from Blueberries Can Inhibit Colon Cancer Cell Proliferation and Induce Apoptosis. Journal of Agricultural and Food Chemistry, 53(18), 7320-7329. 